\title{
BMJ Open Demographic and clinical factors associated with different antidepressant treatments: a retrospective cohort study design in a UK psychiatric healthcare setting
}

\author{
Andrea C Fernandes, ${ }^{1}$ David Chandran, ${ }^{1,2}$ Mizanur Khondoker, ${ }^{3}$ Michael Dewey, ${ }^{4}$ \\ Hitesh Shetty, ${ }^{2}$ Rina Dutta, ${ }^{1,2}$ Robert Stewart ${ }^{1,2}$
}

To cite: Fernandes AC, Chandran D, Khondoker M, et al. Demographic and clinical factors associated with different antidepressant treatments: a retrospective cohort study design in a UK psychiatric healthcare setting. BMJ Open 2018;8:e022170. doi:10.1136/ bmjopen-2018-022170

- Prepublication history and additional material for this paper are available online. To view these files, please visit the journal online (http://dx.doi org/10.1136/bmjopen-2018022170).

Received 18 March 2018 Revised 28 May 2018 Accepted 1 August 2018

Check for updates

(C) Author(s) (or their employer(s)) 2018. Re-use permitted under CC BY-NC. No commercial re-use. See rights and permissions. Published by BMJ.

${ }^{1}$ Institute of Psychiatry, Psychology and Neuroscience, King's College London (KCL), London, UK

${ }^{2}$ South London and Maudsley NHS Foundation Trust, London, UK

${ }^{3}$ Department of Medical Statistics, University of East Anglia, Norwich, UK

${ }^{4}$ Freelance Health Statistics Consultant and KCL, London, UK

Correspondence to

Andrea C Fernandes;

andrea.fernandes@kcl.ac.uk

\section{ABSTRACT}

Objective To investigate the demographic and clinical factors associated with antidepressant use for depressive disorder in a psychiatric healthcare setting using a retrospective cohort study design.

Setting Data were extracted from a de-identified data resource sourced from the electronic health records of a London mental health service. Relative risk ratios (RRRs) were obtained from multinomial logistic regression analysis to ascertain the probability of receiving common antidepressant treatments relative to sertraline.

Participants Patients were included if they received mental healthcare and a diagnosis of depression with antidepressant treatment between March and August 2015 and exposures were measured over the preceding 12 months.

Results Older age was associated with increased use of all antidepressants compared with sertraline, except for negative associations with fluoxetine (RRR $0.98 ; 95 \% \mathrm{Cl}$ 0.96 to 0.98 ) and a combination of two selective serotonin reuptake inhibitors (SSRIs) $(0.98 ; 95 \% \mathrm{Cl} 0.96$ to 0.99$)$, and no significant association with escitalopram. Male gender was associated with increased use of mirtazapine compared with sertraline $(2.57 ; 95 \% \mathrm{Cl} 1.85$ to 3.57$)$. Previous antidepressant, antipsychotic and mood stabiliser use were associated with newer antidepressant use (ie, selective norepinephrine reuptake inhibitors, mirtazapine or a combination of both), while affective symptoms were associated with reduced use of citalopram $(0.58$; $95 \% \mathrm{Cl} 0.27$ to 0.83$)$ and fluoxetine $(0.42 ; 95 \% \mathrm{Cl} 0.22$ to 0.72 ) and somatic symptoms were associated with increased use of mirtazapine $(1.60 ; 95 \% \mathrm{Cl} 1.00$ to 2.75$)$ relative to sertraline. In patients older than 25 years, past benzodiazepine use was associated with a combination of SSRls (2.97; 95\% Cl 1.32 to 6.68), mirtazapine (1.94; $95 \% \mathrm{Cl} 1.20$ to 3.16$)$ and venlafaxine $(1.87 ; 95 \% \mathrm{Cl} 1.04$ to 3.34 ), while past suicide attempts were associated with increased use of fluoxetine $(2.06 ; 95 \% \mathrm{Cl} 1.10$ to 3.87$)$ relative to sertraline.

Conclusion There were several factors associated with different antidepressant receipt in psychiatric healthcare. In patients aged $>25$, those on fluoxetine were more likely to have past suicide attempt, while past use of antidepressant and non-antidepressant use was also
Strengths and limitations of this study

- To our knowledge, this is the first study profiling correlates of antidepressant use in a cohort of patients receiving treatment in a mental health service setting in the UK.

- The data included hard to measure variables such as depressive symptoms, derived using text mining algorithms.

- Focusing on antidepressant data in a 6-month window limited sample sizes for some analyses.

- The analysis is cross-sectional and causal relationships cannot be inferred from associations observed.

- The duration of the antidepressant treatment before and during the observation period was not known.

associated with use of new generation antidepressants, potentially reflecting perceived treatment resistance.

\section{INTRODUCTION}

There are standard national guidelines directing antidepressant prescription in secondary care $^{1}$ (secondary care refers to non-first-line treatment services provided by health professionals to patients who are usually referred by their primary care provider such as general practitioner). British guidelines for the management of depression in secondary care specify that care plans should involve, among other things, the development of a crisis plan that identifies and details management of potential triggers for a depressive episode or a worsening of existing symptoms. ${ }^{1}$ However, in practice a range of patient and clinician characteristics may influence antidepressant prescription. ${ }^{12}$ For example, a community-based study of data collected from 10 psychiatrists who offered antidepressant treatment to 1137 patients found that this 
was influenced by avoidance of side effects, the presence of comorbidities and the presence of specific depressive symptoms such as anxiety, insomnia, fatigue, irritability or increased appetite. ${ }^{3}$ Supporting these results, studies profiling antidepressant prescriptions have established that patient-clinician relationships, past experiences with medication and patient symptoms can influence antidepressant prescription; however, to date these have been set in primary care. ${ }^{4-7}$

There are few studies investigating factors associated with antidepressant use in a secondary mental healthcare setting, ${ }^{89}$ where antidepressant use is common, although more frequently in cases where first-line treatment has been ineffective or is complex for other reasons. ${ }^{10}$ The limited availability of secondary mental healthcare (compared with primary care) data for research, ${ }^{11}{ }^{12}$ has presented wider challenges for the identification of treatment receipt and response, ${ }^{13}$ compounded by the limited availability of data on important clinical parameters such as depressive symptoms ${ }^{14}$ due to variation in clinical recording practices. ${ }^{15}$ Limited data availability can be countered by novel approaches to information extraction applied to health records databases. For example, natural language processing techniques are being introduced in psychiatric research to help with data extraction on varying levels. ${ }^{16}$ In a systematic review, Abbe et $a l^{16}$ identified 38 studies using natural language processing techniques for psychiatry-related research, including analyses of patient perspectives on diagnosis and treatment, detecting diagnosis based on frequency of use of relevant terms, analyses of medical literature and analyses of psychiatric clinical records. The review emphasised the potential value of text mining but also the unique challenges faced in this field, such as the mentions of emotions and subtle descriptions of personality or characteristics which may indicate symptomatology, the challenge of distinguishing of terms with multiple meanings and the requirement for very large training corpora to achieve robust results.

To the best of our knowledge, there has not been a study profiling antidepressant prescription in the UK, outside of primary care. ${ }^{17} 18$ The objective of our study was to investigate whether clinical symptoms (extracted with help of text mining techniques), past antidepressant or other psychotropic treatment or demographic factors are associated with different antidepressant treatment schedules used for depressive disorders in a secondary psychiatric healthcare setting.

\section{METHODS}

\section{The dataset}

The Clinical Record Interactive Search (CRIS) system provides de-identified case note information from the South London and Maudsley (SLaM) National Health Service Trust, a large mental healthcare provider serving a geographical catchment of approximately 1.3 million residents in four South London boroughs (Lambeth, Southwark, Lewisham and Croydon). Electronic health records have been used comprehensively across all SLaM services since 2006. CRIS was established in 2008 to allow searching and retrieval of de-identified ${ }^{19}$ clinical information for research purposes within a robust, patient-led governance framework and currently houses records on over 270000 cases. ${ }^{20}{ }^{21}$ The system allows for retrieval of information from de-identified structured and free-text fields

\section{Observation window and inclusion criteria}

A 6-month interval from 1 March to 31 August 2015 was selected as the observation period where the type of antidepressant use, defined as single antidepressant use and antidepressants users on two antidepressants (referred to here as binary antidepressant use) represented the main dependent variable. All antidepressant use groups were mutually exclusive as described in the next section. Independent variables (demographic and clinical variables) were extracted over the 12 months prior to the observation window and their associations with antidepressant use within the observation window were analysed.

Patients were included if they received active SLaM care within the 6-month observation interval, and if they had received any of the following clinical diagnoses according to 10th Revision of the International Classification of Diseases (ICD) codes on or before the 31 August 2015: depressive episode (F32), recurrent depressive disorder (F33), dysthymia (F34.1), and/or mixed anxiety and depression (F41.2). Individuals were excluded if they had received any F01-F09 (organic disorders), F20-F29 (schizophrenia-like disorders) or F31 (bipolar disorder) diagnoses. Active care was defined as at least one faceto-face contact with SLaM service within the observation window.

\section{Antidepressant use}

Antidepressant use was ascertained using an algorithm developed in General Architecture for Text Engineering (GATE) software, ${ }^{22}$ designed to extract data specifically on any medication recorded in free-text fields (eg, case notes, correspondence). Details of the development of this medication extraction algorithm have previously been published. ${ }^{1421}$ For each patient in the final cohort, any antidepressant use (regardless of the duration of use) during the observation window was extracted as a binary variable. The antidepressants for which data were collected comprised the following: (1) tricyclic and tricyclic related-amitriptyline, clomipramine, dosulepin, doxepin, imipramine, lofepramine, nortriptyline, trimipramine, mianserin and trazodone; (2) monoamine oxidase inhibitors isocarboxazid, phenelzine, tranylcypromine and moclobemide; (3) selective serotonin reuptake inhibitors (SSRIs)-sertraline, fluoxetine, citalopram, escitalopram, fluvoxamine and paroxetine; (4) selective norepinephrine reuptake inhibitors (SNRIs) venlafaxine, reboxetine and duloxetine and (5) Other classes-agomelatine, bupropion and mirtazapine. 
The final cohort comprised 1561 patients. The most common mutually exclusive monotherapy antidepressant used within the 6-month window was sertraline (360 patients), followed by mirtazapine (305), citalopram (213), fluoxetine (200), venlafaxine (143), escitalopram (52). The most common mutually exclusive double antidepressant combinations were any one SSRI with mirtazapine (110), any one SNRI with mirtazapine (89) and a combination of any two SSRIs (89). Groups with no antidepressant use data $(\mathrm{n}=1936)$ were excluded, as were groups with less than 50 patients (to avoid small cell sizes) and those where algorithms indicated use of three or more antidepressants $(n=494)$. All antidepressant groups were mutually exclusive.

\section{Covariates}

Apart from demographic variables, all other covariate data were extracted from data recorded within the 12 months prior to the observation window. Demographic variables were extracted from structured fields, but all other data variables (listed below) were individually extracted using GATE and TextHunter, two text mining software tools which can be programmed to extract data from free-text notes via rules-based and machine learning techniques, respectively. All clinical symptom data variables were extracted using TextHunter.

\section{Demographic variables}

Ethnic group, age, gender, marital status were extracted from structured fields in the source record. Area-level deprivation was measured from the Index of Multiple Deprivation (IMD) that is derived from 2011 national Census data for each individual address, aggregated to Lower Super Output Areas-geographical units with a mean of 1500 inhabitants. The widely used IMD score combines Census-derived data across multiple domains (income, employment, health, education, barriers to housing/services, living environment, crime) and ranks each area at a national level. ${ }^{23}$

\section{Diagnosis-derived depression severity}

Based on the latest-recorded diagnostic code, depression severity was estimated in three categories-mild, moderate/severe and unspecified. Mild severity included diagnoses of F32.0 mild depressive episode, mild, F34.0 recurrent depressive episode, mild, F34.1 dysthymia and F41.2 mixed anxiety and depressive disorders. Moderate to severe depressive severity included diagnoses of F32.1 moderate depressive episode, F32.3 severe depressive episode with psychotic episode, F32.2 severe depression without psychotic episode, F33.1 recurrent depressive episode, current episode moderate, F33.3 and F33.2, respectively, recurrent depressive episode with and without psychotic episode and F33.8 other recurrent depressive, unspecified. Unspecified severity was defined where there was no specified severity of depression within the ICD-code of depression, such as F32 depressive episode (unspecified severity), other depressive episodes, depressive episode, unspecified, F33 recurrent depressive disorder (unspecified severity), F33.4 recurrent depressive in remission (unspecified severity).

\section{Past medication/therapy use}

Antipsychotic, benzodiazepine, mood stabiliser and antidepressant use in the preceding 12 months were ascertained, as were the duration of the current treatment episode, any inpatient in the past 12 months, psychotherapy in the past 12 months and referral to improving access to psychiatric treatment (IAPT) services. IAPT services are a nationwide initiative introduced to increase access to psychological treatments for common mental disorders in primary care.

\section{Clinical symptom data}

As described, bespoke natural language processing algorithms were applied to ascertain symptoms mentioned as present in text fields from the source electronic records. Data on 14 symptoms of depression were extracted on text fields from the preceding 12 months. To avoid small patient groups for analysis, depressive symptoms were categorised into subscales supported by previous results from confirmatory factor analysis on depression scale items (Beck Depressive Inventory-II) in a clinical psychiatric population ${ }^{24}$ and subsequently supported by other findings ${ }^{25}$ although with possible variation in factors at older ages. ${ }^{27}$ Hence the groupings were as follows: Cognitive symptoms: helplessness, worthlessness and hopelessness; Affective symptoms: anhedonia, poor motivation, apathy and low mood; Somatic symptoms: poor concentration, agitation, irritability, low energy, insomnia, poor appetite and anergia. In addition, the presence of psychotic symptoms over the previous 12 months was ascertained from algorithms for hallucinations and delusions. For these symptom groups, in order to minimise false positive occurrences, two or more mentions of symptoms in that domain were classified as a positive instance. Suicidal behaviour was ascertained over the preceding 12 months using natural language processing algorithms for suicidal ideation and suicide attempt.

\section{Patient and public involvement}

Patients and public were not directly involved in this study, although all projects using the CRIS data resource are considered and approved by a patient-led oversight committee. $^{19}$

\section{Statistical analysis}

Group differences were analysed using $\chi^{2}$ tests for categorical variables, using t-tests or one-way analyses of variance for normally distributed continuous variables and using the Kruskal-Wallis test for non-normally distributed continuous data. Multinomial logistic regression analyses were used to answer the research question. The probability of receiving any of the common antidepressant treatments relative to sertraline (the referent, most used, antidepressant category) was estimated using exponentiated regression coefficients from the multinomial 
regression which represents relative risk ratios (RRRs). To build a representative model of which correlates predict antidepressant use in secondary care, decisions to include variables in the final model were guided by the association of each variable in an initial model including age and gender as covariates. Variables that did not have any significant associations in age-adjusted and gender- adjusted analysis were not included in the final model. For initial selection based on age-adjusted and gender-adjusted analysis, the significance was set at $\mathrm{p} \leq 0.25 .^{28}$ This was to help minimise (1) exclusion of potentially key covariates and (2) inclusion of $\mathrm{p}<0.05$ significant estimates occurring by chance. The selected variables were then simultaneously entered in a full model. In the fully adjusted model, significance level was set at $\mathrm{p}<0.05$ for retaining variables. The correlates retained in the final multinomial regression model were age, gender, past inpatient status, past benzodiazepine use, past antipsychotic use, past mood stabiliser use, past antidepressant use, past psychotherapy and IAPT referrals, past experience of psychotic, somatic, affective and cognitive symptoms; and past experience of suicidal ideation and past suicide attempts. In addition, separate analysis was conducted in those aged 26 and over, on the assumption that different factors may be taken into consideration when considering prescriptions of antidepressants to children, adolescents and young people relative to adults. ${ }^{129}$

Any missing values were treated as null values in the analysis as they formed less than $5 \%$ of data. As part of sensitivity analysis, and to ensure important variables are not being excluded in the final model, a full multivariable model was analysed and any variables that were statistically and clinically non-significant were then removed from the model. The results from the final models (from the entire cohort analysis and the adult-only cohort) are available in online supplementary appendix for comparison.

\section{RESULTS}

Table 1 compares patient characteristics between patients using and not using antidepressants. Groups differed in age, ethnicity and deprivation with patients on antidepressants being older, less deprived and of white background. Table 2 compares each antidepressant or antidepressant combination group by demographics, referral data, past medication use and past psychological therapy referral. The demographic and clinical differences between the antidepressant groups are statistically significant apart from ethnicity, area-level deprivation and having had a past referral for psychotherapy. Notably, patients receiving fluoxetine were younger, while older patients were over-represented in those receiving a combination of newer antidepressants such as mirtazapine and an SNRI; there were more males receiving mirtazapine than females; most of the antidepressant users were single, and only a minority had recorded non-antidepressant psychotropic treatments or psychotherapy in the past. Table 3 indicates that the majority of patients had a moderate to

Table 1 Comparing characteristics between patients receiving or not receiving antidepressants during the 6-month evaluation period $(n=3497)$

\begin{tabular}{|c|c|c|c|}
\hline Patient characteristics & $\begin{array}{l}\text { On single or dual therapy } \\
\text { antidepressants } n=1561\end{array}$ & $\begin{array}{l}\text { Not on } \\
\text { antidepressants } n=1936\end{array}$ & \\
\hline \multicolumn{4}{|l|}{ Gender } \\
\hline Male & 706 & 759 & \\
\hline Mean age & 44.3years & 40.1 years & $t=-7.6, d f=3908, p<0.001$ \\
\hline Married & $456(23.5 \%)$ & $319(15.5 \%)$ & \\
\hline Other & $389(20.0 \%)$ & $434(21.1 \%)$ & \\
\hline \multicolumn{4}{|c|}{ Area-level deprivation score tertile } \\
\hline 2.25-22.3 (least deprived) & $631(33.0 \%)$ & $580(28.7 \%)$ & $x^{2}=9.1, d f=2, p<0.05$ \\
\hline White & $1220(63.9 \%)$ & $864(42.1 \%)$ & $x^{2}=10.5, d f=1, p<0.01$ \\
\hline Other & $716(36.1 \%)$ & $1191(57.9 \%)$ & \\
\hline \multicolumn{4}{|c|}{ Depression severity from diagnosis } \\
\hline Mild & $383(24.5 \%)$ & $460(28.3 \%)$ & $x^{2}=0.007, d f=2, p=0.99$ \\
\hline Moderate to severe & $845(54.1 \%)$ & 845 (48.4\%) & \\
\hline Unspecified & $333(21.3 \%)$ & $379(23.3 \%)$ & \\
\hline
\end{tabular}




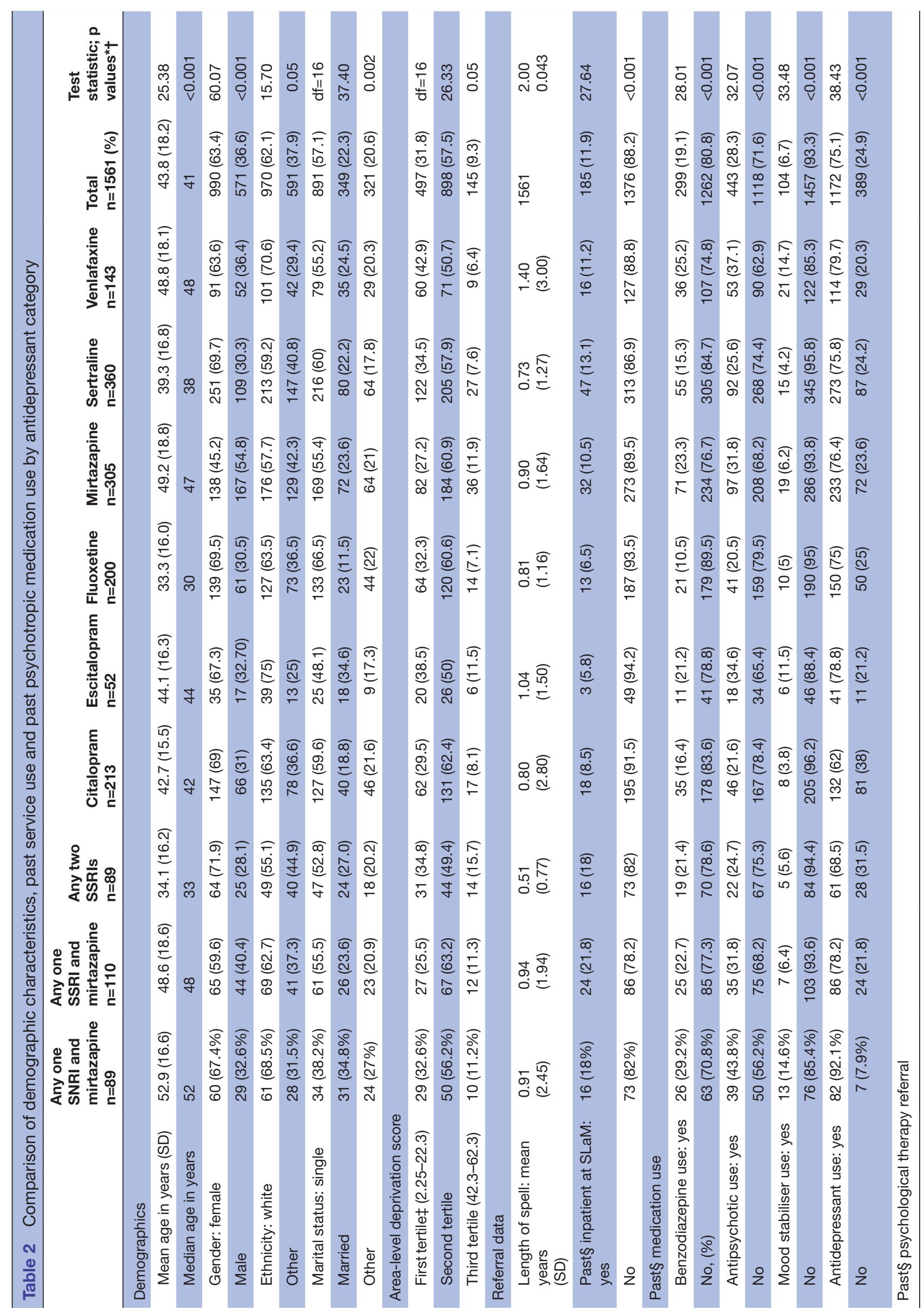


severe diagnosis of depression, a large majority of whom were not recorded as having experienced suicidal ideation or attempted suicide in the preceding 12 months. Apart from diagnostic severity and past psychotic symptoms, for all other past depressive symptoms and past suicidal ideation and attempts, the analysis indicated significant heterogeneity across the antidepressant user groups.

Tables 4 and 5 show the RRR, estimating the probability of patients being on any antidepressant relative to sertraline (the most common antidepressant received in our cohort), derived from the fully adjusted multinomial logistic regression models in the entire cohort and the adult-only cohort, respectively. Table 4 shows that relative to females, males are more likely to be on mirtazapine relative to sertraline. It also indicates that patients on newer antidepressants are more likely to have used non-antidepressant psychotropic medication or been on antidepressants in the past. Those on citalopram or fluoxetine are less likely to have experienced affective symptoms (such as low mood and poor motivation) in the past compared with those on sertraline, while those who have experienced somatic symptoms (such as insomnia and agitation) in the past are more likely to be on mirtazapine relative to sertraline. In the entire cohort and in those aged $>25$, older age was associated with higher probability of being prescribed new generation antidepressants, namely-a combination of any one SNRI and mirtazapine, a combination of any one SSRI and mirtazapine, mirtazapine, and venlafaxine compared with sertraline. In the entire cohort older age was associated with a decreased probability of being prescribed any one SSRI (tables 4 and 5) compared with sertraline.

The results from the analysis in those aged $>25$ (table 5) show increased likelihood of males being on mirtazapine compared with females. They also show that, relative to sertraline, patients on citalopram or fluoxetine are less like to have recorded affective symptoms, patients on mirtazapine are more likely to have recorded somatic symptoms and patients on fluoxetine were more likely to have a recorded suicide attempt in the past 12 months relative to those on sertraline. The sensitivity analyses did not give rise to any marked difference in findings (see online supplementary appendix).

\section{DISCUSSION}

The aim of this study was to ascertain patient demographic and clinical factors that correlate with different antidepressant treatments in patients actively receiving secondary mental healthcare for clinical depression. While there were no strongly consistent trends across all comparison groups or exposures, our results suggest that age, past medication and/or psychotherapy receipt use and symptom profiles in the past 12 months have potentially some influence on antidepressant receipt in secondary mental healthcare.

We found that older patients were less likely to be using fluoxetine or a combination of two SSRIs relative to 


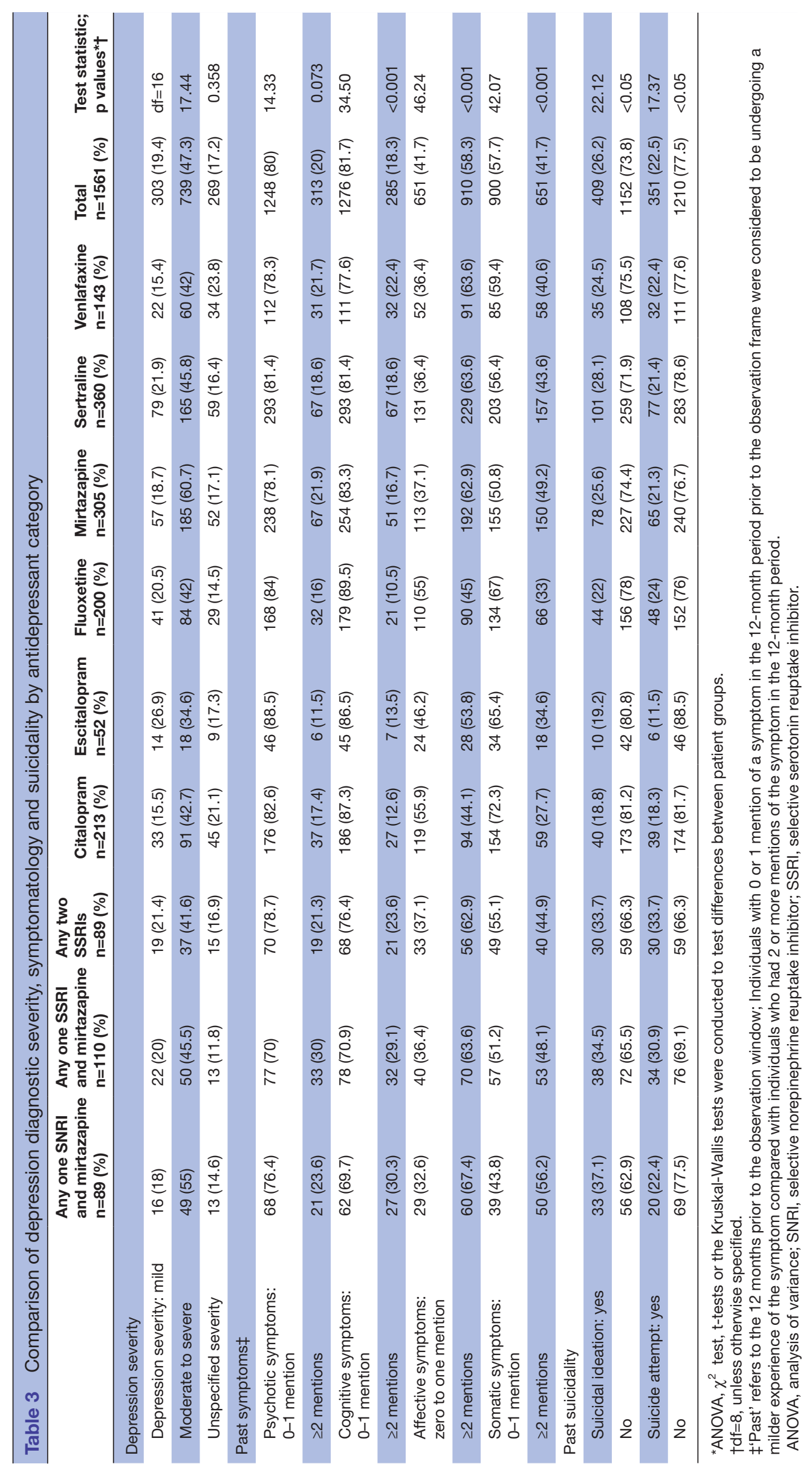




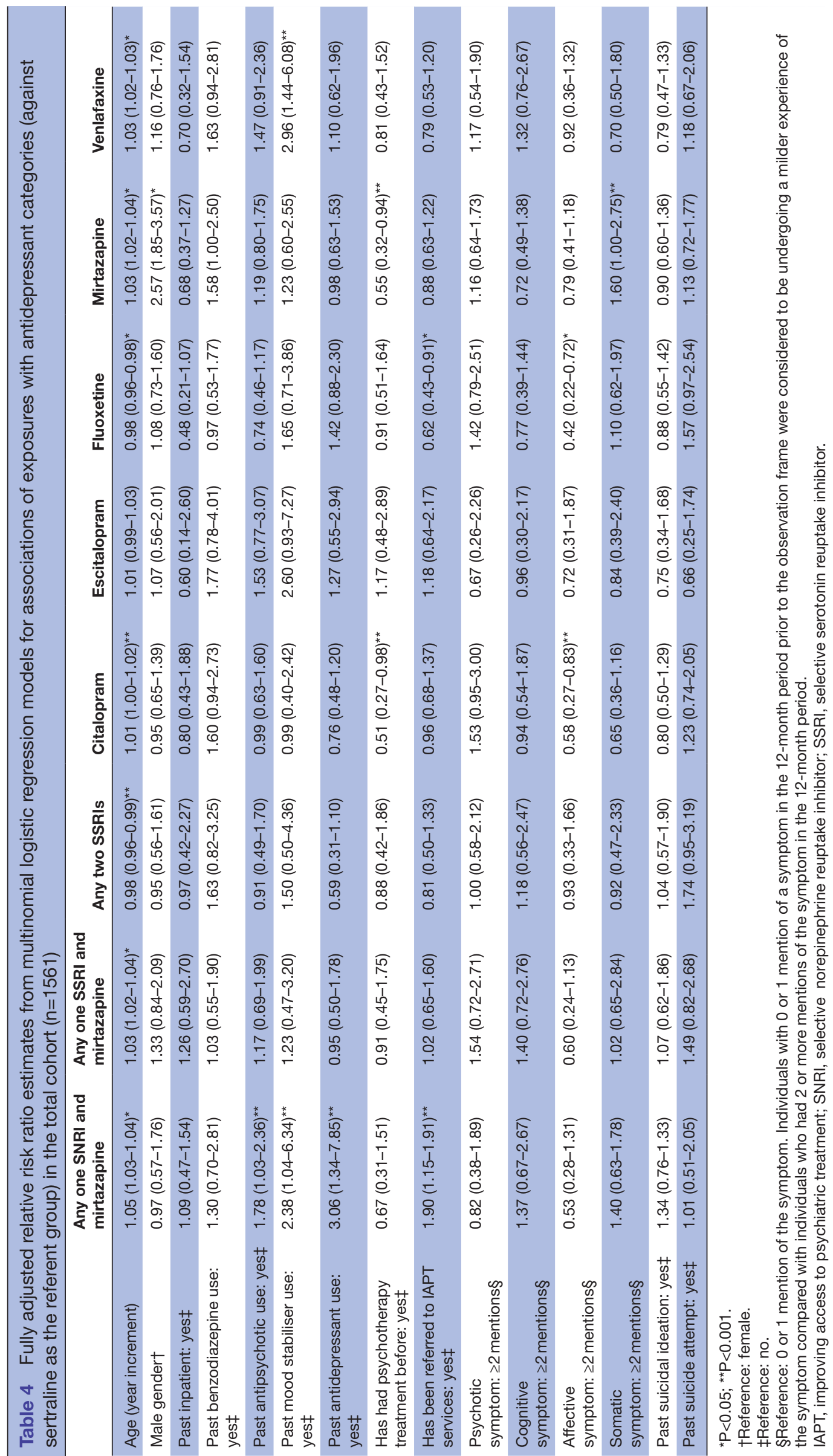




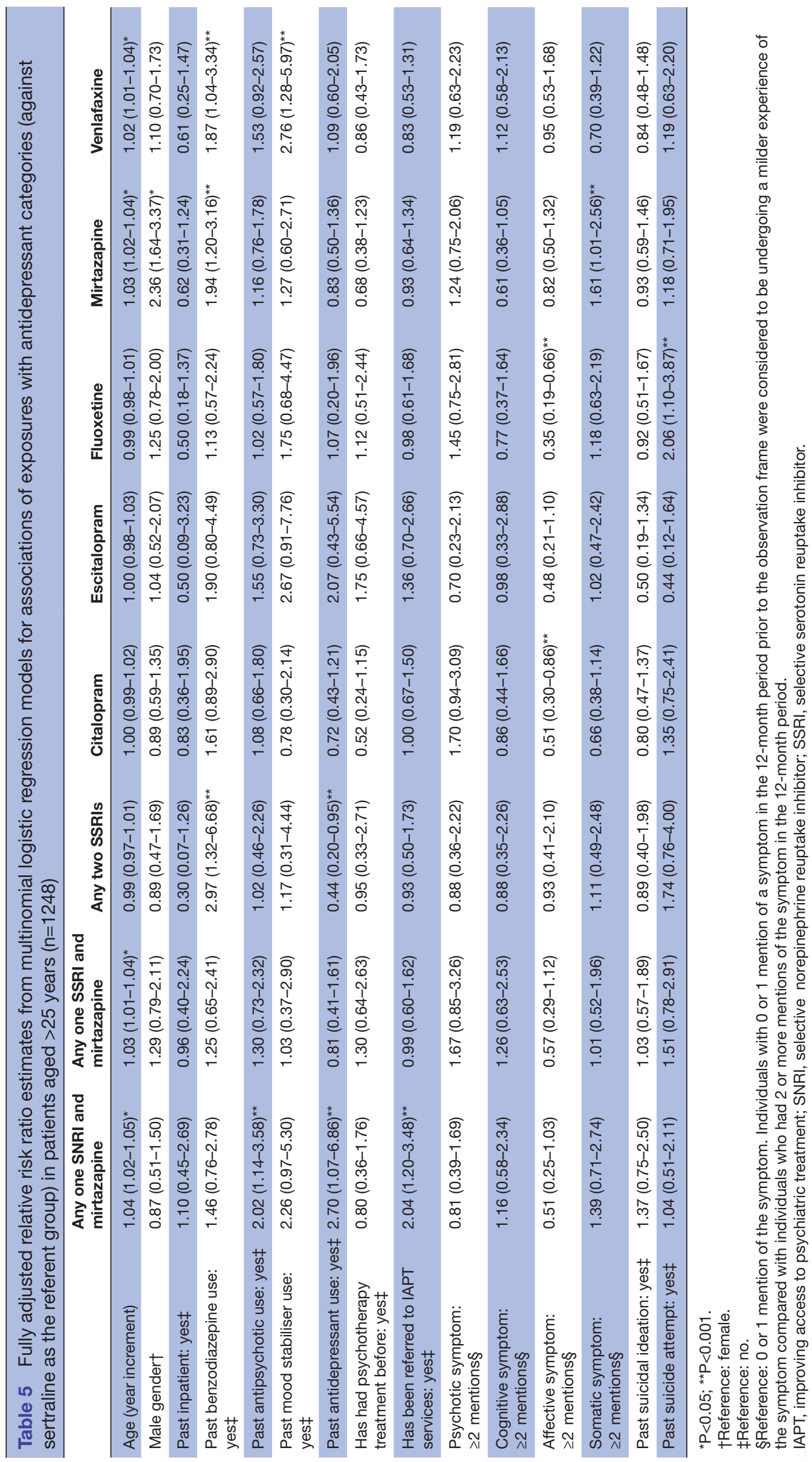


sertraline and were more likely to be on other combinations, citalopram, mirtazapine or venlafaxine. Comparable to our findings, a study of newly initiated antidepressant use in a cohort of over 500000 war veterans with a diagnosis of depression ${ }^{9}$ found that older patients were less likely (RRR 0.70) to have started on fluoxetine than sertraline. While we did not seek to collect data on the reasoning behind the choices, although this might reflect concerns around the longer half-life of fluoxetine in older age groups and the lower propensity of sertraline for cytochrome-related interactions with coprescribed medications, the association was less strong in the subgroup aged $>25$, so might be more likely to reflect lower perceived risk of treatment-emergent suicidality associated with fluoxetine in adolescents and younger adults. Similarly, the association of older age with mirtazapine use, individually or in combination, may reflect perceived likelihood of sedation and weight gain-considered problematic in younger patients but potentially advantageous (when taken at night) in late life depression. On the other hand, the higher use of mirtazapine (compared with sertraline) in men compared with women may reflect lower incidence of sexual dysfunction side effects. ${ }^{30}$ Of interest, we found no substantial variation in use by ethnicity, marital status or neighbourhood socioeconomic status, once age and gender had been accounted for, suggesting little evidence of socially determined variation in prescribing.

Considering past medication use, antipsychotic, mood stabiliser or antidepressant use in the previous 12 months was associated with a higher likelihood of the co-occurrence of SNRI with mirtazapine during the observation period, and mood stabiliser use was also predictive of venlafaxine compared with sertraline use. The SNRImirtazapine group were also more likely to have been referred for primary care based psychotherapy (the UK's IAPT service model). As this secondary care cohort is likely to have an over-representation of individuals with some resistance to first-line antidepressant treatment, it may follow that these individuals were on other treatment regimens in the past and that newer antidepressant regimens were being prescribed in the context of higher levels of treatment resistance. However, it is hard to define, and therefore identify, patients who are resistant to antidepressant treatment especially from naturalistic clinical databases, so conclusions can only be tentative.

Considering clinical features, suicidal ideation and past suicide attempts were not significantly associated with different antidepressant use overall; however, past suicide attempt was associated with an increased likelihood of fluoxetine compared with sertraline receipt in patients aged $>25$ which, as discussed above for age effects, may reflect the evidence collated from meta-analyses of randomised controlled trials and observation studies where fluoxetine has been shown to carry reduced risk of emergent suicidal behaviour in adults compared with children and adolescents. ${ }^{31-33}$ Those with affective symptoms recorded were less likely to be on citalopram or fluoxetine than sertraline, while those with somatic symptoms were more likely to be on mirtazapine, associations which persisted in the cohort aged $>25$. Clinician prescribing preference may be influenced by perceived therapeutic actions or perceived risk of adverse drug events, or both. For example, the somatic symptoms of poor appetite and insomnia may increase the likelihood of a medication such as mirtazapine being prescribed because of recognised propensity to sedation and weight gain. There is some evidence to suggest citalopram may induce cardiotoxicity in overdose ${ }^{34}$; however, apart from clinical guidelines on when to prescribe antidepressants studies are rarely conducted to assess the association singular drugs with depressive symptoms are being prescribed. ${ }^{35}$

As mentioned, there has been very little previous research into factors associated with different antidepressant treatments for depression, particularly in secondary care. Improved knowledge in this area is important for several reasons. First, it is helpful to understand factors potentially influencing prescribing behaviour to assess the impact of guidelines and to describe variations beyond guidelines. From our observations here, the relative lack of covariates consistently associated with antidepressant use suggests prescribing based on individual experiences after patient-clinician communication, consistent with national guidelines emphasising patientclinician communication. ${ }^{35}$ However, findings suggestive of prescribing motivations derived from observational data of this nature need further qualitative studies for confirmation. Second, while variations in prescribing may reflect potentially problematic non-evidence-based behaviour, it might also highlight novel patterns arising from clinical experience which need to be noted and assessed further to improve the evidence base. Early detection of adverse or unexpectedly beneficial, effects is an example of these indications of how antidepressants are being used for treatment in secondary care is more realistic than research from pharmaceutical trials. Our findings suggest that there are certain antidepressants that are avoided in certain clinical scenarios; for example, patients previously described as having symptoms in the 'affective' category were less likely to be receiving citalopram or fluoxetine compared with sertraline. There is a suggestion from the results that newer generation antidepressants are used to treat more severe depression which may indicate greater clinician-perceived tolerability and/ or lower toxicity and/or lower propensity to interact with other coprescribed medication. ${ }^{29} 35$

The results should be interpreted with study limitations in mind. First, using antidepressant data in a 6-month window limited sample sizes for analysis, and inaccurate measures of exposure status (eg, the use of area-level rather than individual-level socioeconomic status) might have reduced the likelihood of identifying underlying associations. Second, the study is close to cross-sectional design, since there may have been co-occurrence of exposures and outcomes in the prior 12-month observation period; therefore, direct causal relationships cannot be 
conclusively inferred. Third, the duration of the antidepressant treatment during the observation period was not known, so some patients will have been new users of antidepressants identified, while others may have been using the antidepressant(s) for much longer periods of time; similarly, where there was co-occurrence of two antidepressants during the time period evaluated, it was not possible to distinguish a switch from one agent to another from coprescribing of the two agents. Fourth, the results of the study cannot assumed to be generalisable to all patients in secondary mental healthcare settings due to the social and ethnic diversity found in Southeast London from where this sample was drawn; in addition, the focus of the study was on antidepressant use in people with a depressive disorder diagnosis and being reviewed in secondary mental healthcare, so findings cannot assumed to generalise to antidepressant use in other circumstances or for other indications. Finally, we could only investigate factors that were available to us in the current dataset. The literature reports various clinical covariates, ${ }^{8}$ patient demographics, ${ }^{9}$ medication use, ${ }^{36}$ clinician characteristics $^{3}$ and clinical location ${ }^{89}$ as factors involved for antidepressant prescription in secondary mental healthcare settings. We could not capture all these factors in our analysis.

Limitations notwithstanding there are key strengths to this paper. To our knowledge, this is the first study profiling antidepressant use in a cohort of patients receiving treatment for depression in a secondary mental healthcare setting in the UK. Although from a single site, the use of health records data provides a more generalisable sample than would be possible through a conventional cohort design involving de novo interviews, let alone through the even more selected samples in clinical trials. In addition, the data included constructs such as depressive symptoms that are not usually available in administrative 'big' data, taking advantage of a suite of recently developed text mining algorithms to capture a greater depth of data from free-text fields, circumventing the usual restriction of analysed data to those recorded in structured fields. The findings have the potential to inform clinical practice within this clinical setting. Knowledge of factors involved in antidepressant prescription could be used to audit clinical practices and inform whether the clinical practices are benefiting patient treatment outcomes. ${ }^{37}$ However, further qualitative work is definitely indicated to identify and highlight processes involved in clinician selection of antidepressant treatment. Continual monitoring of treatment choices in this cohort may contribute to providing optimal care for secondary care patients and there may be scope for further quantitative evaluation across a wider range of services, and evaluating longer term outcomes associated with different treatment decisions.

Acknowledgements The authors of this work thank MD for initial advice and guidance on the statistical analysis, and extend sincere gratitude to MK for his patient and continuous support on statistical analysis.
Contributors RS, RD and ACF conceived of the study and were responsible for the design and search strategy. ACF, HS and DC were responsible for data extraction. ACF was responsible for conducting the analysis and producing the tables. The analysis was guided by MD initially and then MK. The initial draft of the manuscript was prepared by ACF then circulated among all authors for revision. All authors helped to evolve analysis plans, interpret data and critically revise successive drafts of the manuscript.

Funding The National Institute for Health Research (NIHR) Biomedical Research Centre at South London and Maudsley NHS Foundation Trust, King's College London and Mental Health Research UK. DC, HS, RD and RS are part funded by the National Institute for Health Research (NIHR) Biomedical Research Centre at South London and Maudsley NHS Foundation Trust and King's College London. ACF is funded by a Mark Robinson studentship awarded by Mental Health Research UK.

Disclaimer The lead author (the manuscript's guarantor) affirms that the manuscript is an honest, accurate and transparent account of the study being reported; that no important aspects of the study have been omitted and that any discrepancies from the study as planned have been explained.

Competing interests None declared.

Patient consent Not required.

Ethics approval The use of CRIS for research was approved by the 0xfordshire Research Ethics Committee C (reference 08/H0606/71+5).

Provenance and peer review Not commissioned; externally peer reviewed.

Data sharing statement No additional data are available.

Open access This is an open access article distributed in accordance with the Creative Commons Attribution Non Commercial (CC BY-NC 4.0) license, which permits others to distribute, remix, adapt, build upon this work non-commercially, and license their derivative works on different terms, provided the original work is properly cited, appropriate credit is given, any changes made indicated, and the use is non-commercial. See: http://creativecommons.org/licenses/by-nc/4.0/.

\section{REFERENCES}

1. National Institute for Health \& Clinical Excellence (NICE) Guideline. National clinical practice guideline 90: depression the NICE guidelines on the treatment and management of depression in adults - the updated edition: The British Psychological Society and The Royal College of Psychiatrists, 2010.

2. Zetin M, Hoepner CT, Bjornson L. Rational antidepressant selection: applying evidence-based medicine to complex real-world patients. Psychopharmacol Bull 2006;39:38-104.

3. Zimmerman M, Posternak M, Friedman M, et al. Which factors influence psychiatrists' selection of antidepressants? Am J Psychiatry 2004;161:1285-9.

4. Meijer WE, Heerdink ER, Leufkens HG, et al. Incidence and determinants of long-term use of antidepressants. Eur J Clin Pharmacol 2004;60:57-61.

5. Sleath B, Shih YC. Sociological influences on antidepressant prescribing. Soc Sci Med 2003;56:1335-44.

6. Bauer M, Monz BU, Montejo AL, et al. Prescribing patterns of antidepressants in Europe: results from the Factors Influencing Depression Endpoints Research (FINDER) study. Eur Psychiatry 2008;23:66-73.

7. Garrison GD, Levin GM. Factors affecting prescribing of the newer antidepressants. Ann Pharmacother 2000;34:10-14.

8. Kim HM, Zivin K, Choe HM, et al. Predictors of start of different antidepressants in patient charts among patients with depression. $J$ Manag Care Spec Pharm 2015;21:424-30.

9. Kim HM, Zivin K, Ganoczy D, et al. Predictors of alternative antidepressant agent initiation among U. S. veterans diagnosed with depression. Pharmacoepidemiol Drug Saf 2010;19:1049-56.

10. Huxley P, Goldberg D. Mental health in the community: the pathways to psychiatric care: London Tavistock Publ, 1980.

11. Weiskopf NG, Hripcsak G, Swaminathan S, et al. Defining and measuring completeness of electronic health records for secondary use. J Biomed Inform 2013;46:830-6.

12. Thiru K, Hassey A, Sullivan F. Systematic review of scope and quality of electronic patient record data in primary care. $B M J$ 2003;326:1070-5.

13. Kashner TM. How good are observational studies in assessing psychiatric treatment outcomes? Am J Psychiatry 2012;169:244-7. 
14. Jackson MSc RG, Ball M, Patel R, et al. Texthunter--a user friendly tool for extracting generic concepts from free text in clinical research. AMIA Annu Symp Proc 2014;2014:729-38.

15. Mathioudakis A, Rousalova I, Gagnat AA, et al. How to keep good clinical records. Breathe 2016;12:369-73.

16. Abbe A, Grouin C, Zweigenbaum P, et al. Text mining applications in psychiatry: a systematic literature review. Int J Methods Psychiatr Res 2016;25:86-100.

17. Mars B, Heron J, Kessler D, et al. Influences on antidepressant prescribing trends in the UK: 1995-2011. Soc Psychiatry Psychiatr Epidemiol 2017;52:193-200.

18. Shi N, Durden E, Torres A, et al. Predictors of treatment with duloxetine or venlafaxine XR among adult patients treated for depression in primary care practices in the United Kingdom. Depress Res Treat 2012;2012:1-8.

19. Fernandes AC, Cloete D, Broadbent MT, et al. Development and evaluation of a de-identification procedure for a case register sourced from mental health electronic records. BMC Med Inform Decis Mak 2013;13:1-14.

20. Stewart R, Soremekun M, Perera G, et al. The South London and Maudsley NHS Foundation Trust Biomedical Research Centre (SLAM BRC) case register: development and descriptive data. BMC Psychiatry 2009;9:51.

21. Perera G, Broadbent M, Callard F, et al. Cohort profile of the South London and Maudsley NHS Foundation Trust Biomedical Research Centre (SLaM BRC) Case Register: current status and recent enhancement of an Electronic Mental Health Record-derived data resource. BMJ Open 2016;6:e008721-2.

22. Cunningham $\mathrm{H}$, Maynard $\mathrm{D}$, Bontcheva $\mathrm{K}$, et al. Text processing with GATE (Version 6): University of Sheffield Department of Computer Science, 2011.

23. DCLG D for $\mathrm{C}$ and LG. The english index of multiple deprivation, 2015.

24. Arnarson TO, Olason DT, Smári J, et al. The Beck Depression Inventory Second Edition (BDI-II): psychometric properties in Icelandic student and patient populations. Nord J Psychiatry 2008;62:360-5.

25. Ward LC. Comparison of factor structure models for the Beck Depression Inventory--II. Psychol Assess 2006;18:81-8.
26. Subica AM, Fowler JC, Elhai JD, et al. Factor structure and diagnostic validity of the beck depression inventory-II with adult clinical inpatients: comparison to a gold-standard diagnostic interview. Psychol Assess 2014;26:1106-15.

27. Prince MJ, Reischies F, Beekman AT, et al. Development of the EURO-D scale--a European, Union initiative to compare symptoms of depression in 14 European centres. Br J Psychiatry 1999;174:330-8.

28. Bursac Z, Gauss $\mathrm{CH}$, Williams DK, et al. Purposeful selection of variables in logistic regression. Source Code Biol Med 2008;3:17.

29. Cleare A, Pariante CM, Young AH, et al. Evidence-based guidelines for treating depressive disorders with antidepressants: A revision of the 2008 British Association for Psychopharmacology guidelines. $J$ Psychopharmacol 2015;29:459-525.

30. Saiz-Ruiz J, Montes JM, Ibáñez A, et al. Assessment of sexual functioning in depressed patients treated with mirtazapine: a naturalistic 6-month study. Hum Psychopharmacol 2005;20:435-40.

31. Barbui C, Esposito E, Cipriani A. Selective serotonin reuptake inhibitors and risk of suicide: a systematic review of observational studies. CMAJ 2009;180:291-7.

32. Gibbons RD, Brown $\mathrm{CH}$, Hur $\mathrm{K}$, et al. Suicidal thoughts and behavior with antidepressant treatment: reanalysis of the randomized placebocontrolled studies of fluoxetine and venlafaxine. Arch Gen Psychiatry 2012;69:580-7.

33. Whittington CJ, Kendall T, Fonagy P, et al. Selective serotonin reuptake inhibitors in childhood depression: systematic review of published versus unpublished data. Lancet 2004;363:1341-5.

34. Cleare A, Pariante CM, Young AH, et al. Evidence-based guidelines for treating depressive disorders with antidepressants: a revision of the 2008 British Association for Psychopharmacology guidelines. $J$ Psychopharmacol 2015;29:459-525.

35. NHS. Formulary and prescribing guidelines: providing partnership services in bedfordshire. Essex and Luton, 2011:1-12.

36. Sirey JA, Meyers BS, Bruce ML, et al. Predictors of antidepressant prescription and early use among depressed outpatients. Am J Psychiatry 1999;156:690-6.

37. Lin SY, Stevens MB. The symptom cluster-based approach to individualize patient-centered treatment for major depression. J Am Board Fam Med 2014;27:151-9. 